Mens

revue d'histoire intellectuelle de l'Amérique française

\title{
Entre nationalisme et socialisme : Raoul Roy (1914-1996) et les origines d'un premier indépendantisme socialiste au Québec, 1935-1965
}

\section{Mathieu Lapointe}

Volume 8, numéro 2, printemps 2008

URI : https://id.erudit.org/iderudit/1022834ar

DOI : https://doi.org/10.7202/1022834ar

Aller au sommaire du numéro

Éditeur(s)

Centre de recherche en civilisation canadienne-française

ISSN

1492-8647 (imprimé)

1927-9299 (numérique)

Découvrir la revue

Citer cet article

Lapointe, M. (2008). Entre nationalisme et socialisme : Raoul Roy (1914-1996) et les origines d'un premier indépendantisme socialiste au Québec, 1935-1965.

Mens, 8(2), 281-322. https://doi.org/10.7202/1022834ar
Résumé de l'article

Contribution à une histoire approfondie des débuts de l'indépendantisme, cet article présente l'étonnant parcours idéologique de Raoul Roy (1914-1996), fondateur des deux premières institutions de l'indépendantisme de gauche, $L a$ Revue socialiste (1959-1965) et l'Action socialiste pour l'indépendance du Québec (ASIQ, 1960-C.1963). Jeune autodidacte campagnard ballotté par la Crise, Roy se dit « séparatiste » à la fin des années 1930 et prône le fascisme comme moyen de libération des Canadiens français, tout en étant attiré par le communisme. Militant après la Deuxième Guerre mondiale dans le mouvement communiste (Parti ouvrier-progressiste) sans nécessairement avoir à renier son nationalisme, il fondera à la fin des années 1950 l'indépendantisme de gauche en élaborant une première version de l'indépendantisme socialiste de décolonisation, dans laquelle le nationalisme prime nettement le socialisme. 
ENTRE NATIONALISME

ET SOCIALISME :

RAOUL ROY (1914-1996) E'T LES

ORIGINES D'UN PREMIER

INDÉPENDANTISME SOCIALISTE

AU QUÉBEC, 1935-1965

\author{
Mathieu Lapointe \\ Département d'histoire \\ Université York
}

\title{
Résumé
}

Contribution à une histoire approfondie des débuts de l'indépendantisme, cet article présente l'étonnant parcours idéologique de Raoul Roy (1914-1996), fondateur des deux premières institutions de l'indépendantisme de gauche, La Revue socialiste (1959-1965) et l'Action socialiste pour l'indépendance du Québec (ASIQ, 1960-c.1963). Jeune autodidacte campagnard ballotté par la Crise, Roy se dit « séparatiste " à la fin des années 1930 et prône le fascisme comme moyen de libération des Canadiens français, tout en étant attiré par le communisme. Militant après la Deuxième Guerre mondiale dans le mouvement communiste (Parti ouvrier-progressiste) sans nécessairement avoir à renier son nationalisme, il fondera à la fin des années 1950 l'indépendantisme de gauche en élaborant une première version de l'indépendantisme socialiste de décolonisation, dans laquelle le nationalisme prime nettement le socialisme. 


\section{Abstract}

This article contributes to the scholarship surrounding Quebec's early independence movement by examining the surprising intellectual itinerary of Raoul Roy (19141996), who founded two of the first institutions of left-wing indépendantisme: the Revue socialiste (1959-1965) and the Action socialiste pour l'indépendance du Québec (ASIQ, 1960-ca. 1963). As a self-taught young rural lad suffering from the effects of the Depression, Roy referred to himself as a "separatist" in the late 1930s and advocated fascism as a tool for the liberation of French Canada. He was also drawn to communism during this era and be joined the Labour-Progressive Party after World War Two. Roy did not renounce nationalism during his time in the communist movement and was instrumental in the emergence of the left-wing independence movement in the late 1950s. He formulated an early version of socialist decolonisation indépendantisme, where socialism was clearly subordinated to nationalism.

\section{Les débuts de l'indépendantisme : une historiographie lacunaire}

Malgré la grande importance qu'a eue le nationalisme québécois, et en particulier le mouvement indépendantiste, dans l'histoire du Québec et du Canada depuis les années 1960, l'historiographie de ces sujets recèle encore de nombreuses et importantes lacunes. Ceci s'explique probablement en bonne partie par la réticence ordinaire de la discipline historique devant des sujets trop récents, les historiens désirant généralement bénéficier, avant de mettre en jeu leur crédibilité professionnelle, du recul des années et d'un accès le plus libre possible au plus grand nombre de sources archivistiques pertinentes.

Quoi qu'il en soit, le fait demeure que la plupart des interprétations classiques de l'essor du nationalisme indépendantiste dans les années 1960 ont été produites par des politologues et des sociologues plutôt que par des historiens. En conséquence, elles insistent surtout, suivant l'habitude de ces 
disciplines, sur les facteurs et les forces macroscopiques (sociologiques, économiques, politiques, idéologiques et culturelles) qui ont permis ou entraîné l'essor de ce mouvement au cours des années 1960, plutôt que sur les personnages qui ont animé le mouvement et milité en son sein ${ }^{2}$. Ou encore - erreur inverse - elles font des politiciens des démiurges et prêtent à leurs gestes un impact démesuré. Ainsi, par exemple, le politologue Dale Thomson attribue-t-il un peu simplement la « faute » de l'essor rapide du nationalisme pendant la Révolution tranquille aux imprudences rhétoriques d'un gouvernement Lesage avide de faire accepter des réformes audacieuses à une population canadienne-française traditionnellement conservatrice : les slogans enflammés (ceux de la campagne de 1962 en particulier) auraient mis le feu aux poudres et déclenché l'incendie que depuis lors on n'a plus réussi à éteindre $^{3}$.

Les interprétations antagoniques des politologues William Coleman et Kenneth McRoberts sont beaucoup plus fouillées et plus riches, mais demeurent néanmoins macroscopiques : McRoberts conçoit la Révolution tranquille et l'essor du nationalisme québécois comme deux phénomènes en continuité et en harmonie, portés par une nouvelle classe moyenne francophone qui serait en émergence depuis la Seconde Guerre mondiale ${ }^{4}$. Coleman prétend au contraire que l'essor du mouvement indépendantiste représente une réaction par rapport à la Révolution tranquille, causée par la rupture de la coalition de classes qui avait permis cette « révolution » et par l'insécurité identitaire et psychologique croissante des classes mécontentes en particulier et de la population canadienne-française en général devant un Québec de plus en plus intégré et semblable au reste de l'Amérique du Nord'. Deux interprétations contraires, porteuses d'intuitions et de questions intéressantes certes, mais demeurant si globales qu'elles ne font qu'effleurer l'histoire proprement dite du 
mouvement indépendantiste ${ }^{6}$. Enfin, le politologue Léon Dion présente quant à lui un portrait plus riche et coloré des mutations de la société québécoise et de l'essor du nouveau nationalisme, mais n'ose pas lui non plus une véritable histoire du mouvement depuis sa naissance en 1957. Tout se passe comme si ces spécialistes des sciences sociales ne s'intéressaient à l'indépendantisme qu'à partir du moment, au cours des années 1960, où celui-ci devient un mouvement social notable, un phénomène important qu'on puisse situer dans un système de facteurs sociaux en évolution.

Par conséquent, il n'existe pas d'histoire satisfaisante, détaillée et érudite, du mouvement indépendantiste d'aprèsguerre à partir de son initiation par Raymond Barbeau en $1957^{\circ}$. Mises à part certaines grandes figures comme René Lévesque, les militants, c'est-à-dire les acteurs historiques qui ont mené ce mouvement, sont en grande partie oubliés dans le récit'. On perd donc une grande part de l'bistoire proprement dite et de ce qu'elle révèle de la texture du réel, sources potentielles de fécondes intuitions d'explication et d'intéressantes hypothèses de recherche.

En outre, même dans les cas où l'on tente effectivement un survol historique de l'indépendantisme d'aprèsguerre, la présentation des débuts du mouvement est généralement superficielle, tronquée et sélective. Peu d'études historiques ont été consacrées à éclairer la genèse et la première décennie de ce mouvement, depuis la fondation en 1957 de l'Alliance laurentienne, premier groupe d'après-guerre voué à l'indépendance du Québec, à celle du Mouvement souveraineté-association (MSA) par René Lévesque en 1967. De plus, la majorité des travaux - comme la quasi-totalité des commémorations - mettent l'accent sur le rôle du Rassemblement pour l'indépendance nationale (RIN), le troisième groupe séparatiste à voir le jour depuis la Deuxième Guerre mondiale 
(en septembre 1960) et celui qui eut le plus de succès au cours de cette première décennie de l'indépendantisme ${ }^{10}$. On tend souvent à faire de la fondation du RIN le moment zéro du mouvement indépendantiste ${ }^{11}$ et l'on relègue aux oubliettes de l'histoire les toutes premières années du mouvement (19571960) ainsi que les deux premiers groupes qui prônèrent l'indépendance du Québec, c'est-à-dire l'Alliance laurentienne de Raymond Barbeau (et sa revue Laurentie) et l'Action socialiste pour l'indépendance du Québec (et $L a$ Revue socialiste) de Raoul Roy ${ }^{12}$. Du côté des revues, on a accordé beaucoup d'attention aux jeunes intellectuels avant-gardistes de la revue Parti pris, qui popularisèrent une esthétique et une pensée nouvelles faisant l'amalgame d'une certaine modernité culturelle, de l'anticléricalisme, du nationalisme indépendantiste, du socialisme et de la théorie de la décolonisation ${ }^{13}$; et l'on a somme toute très peu étudié les premières revues indépendantistes Laurentie et La Revue socialiste. Enfin, de nombreux ouvrages ont été consacrés à l'organisation terroriste du Front de libération du Québec (FLQ), apparu en 1963, qui promouvait lui aussi une idéologie nationaliste socialiste de décolonisation $^{14}$. L'historiographie s'est donc nettement concentrée sur les mouvements et les revues qui eurent le plus de succès ou de visibilité. La conséquence de cette attention sélective est que l'on a jusqu'ici accordé assez peu d'attention à certains personnages de transition, tels l'écrivain Jacques Ferron - du moins à cet égard - et l'intellectuel militant Raoul Roy, qui jouèrent un rôle important dans la transformation du nationalisme québécois au tournant des années 1960 et en particulier dans la formulation et la promotion de l'idéologie indépendantiste socialiste de décolonisation ${ }^{15}$.

Cette absence d'intérêt de l'historiographie pour les personnages qui formulèrent le premier indépendantisme de gauche vient probablement de la nette impression d'une rupture 
profonde entre l'ancien nationalisme et le nouvel indépendantisme. Cette impression s'explique elle-même aisément. Les militants de ces mouvements étaient le plus souvent jeunes et rejetaient violemment le traditionalisme ou la modération de leurs devanciers. Ils professaient une nouvelle doctrine qui radicalisait à plusieurs égards le néo-nationalisme de Laurendeau et des historiens de Montréal. Et ils se situaient en même temps clairement à gauche, se disant même souvent socialistes, alors que la gauche canadienne-française avait auparavant été soit franchement antinationaliste (comme dans le cas des directeurs de Cité libre ${ }^{1}$ ), soit tout au moins quelque peu méfiante vis-à-vis du nationalisme, cette idéologie que Duplessis utilisait pour légitimer son opposition aux mesures sociales les plus progressistes du gouvernement et de l'administration fédérales. À première vue, donc, le récit semble clair : une nouvelle génération entre bruyamment en scène, qui dans sa fougueuse jeunesse veut rompre tant avec la frilosité et la sclérose passéiste de ses devanciers nationalistes qu'avec l'obstination fédéraliste et antinationaliste de l'ancienne gauche libérale et syndicale, et qui élabore pour cela une nouvelle synthèse à partir d'une nouvelle doctrine historique et des idées d'avant-garde contemporaines (socialisme, décolonisation).

Ce récit de rupture et de révolution idéologique par une génération montante n'est pas tout à fait faux. Mais il occulte le fait que cette rupture profonde ne fut pas spontanée et qu'elle ne fut pas uniquement l'œuvre de jeunes intellectuels. Elle a en fait été désirée et elle fut le fruit d'un effort doctrinal et polémique entrepris par des intellectuels et des militants nés avant la génération de Parti pris. En d'autres mots, il y a eu des passeurs qui, issus d'un passé libéral, socialiste et nationaliste, lancèrent l'indépendantisme socialiste qui sera repris et répandu, à partir de 1963 surtout, par les jeunes in- 
tellectuels et militants de Parti pris et de l'avant-garde intellectuelle québécoise des années 1960.

L'histoire de ces passeurs est intéressante parce qu'elle est enracinée dans l'évolution sociale et idéologique du Québec depuis les années trente et parce qu'elle est celle d'individus aux parcours atypiques, échappant souvent aux mouvances les plus étudiées de l'évolution idéologique québécoise (le traditionalisme et l'action catholique, par exemple). Parmi ces personnages à contre-courant, qui ont souvent rué dans les brancards parce qu'ils se trouvaient au milieu de divers camps idéologiques, Jacques Ferron est l'un de ceux qui ont attiré le plus d'attention, à cause de ses riches talents d'écrivain, de sociographe et d'humoriste polémiste, et l'intérêt de son parcours a été souligné ${ }^{17}$. À l'inverse, la trajectoire de son collaborateur, l'intellectuel militant Raoul Roy (1914-1996), qui fonda en 1959 La Revue socialiste (1959-1965) et en 1960 l'Action socialiste pour l'indépendance du Québec (ASIQ), les deux premières institutions de l'indépendantisme de gauche, est très mal connue.

L'historiographie n'a retenu que peu de choses de la biographie de Roy, idéologue assez marginal dont le rôle historique se limite probablement aux années $1959-1963^{18}$. L'on glane ici et là, dans des articles de journaux et les écrits de Ferron, des informations sur ses engagements et son existence d'après-guerre ${ }^{19}$. On retient en général qu'il fut employé, immédiatement après la guerre, dans l'Union des marins canadiens alors qu'il militait dans la mouvance communiste du Parti ouvrier-progressiste (P.O.P.) ; qu'il quitta ce mouvement à l'instar de nombreux militants canadiens-français lors de la crise qui secoua ce parti en 1947 ; qu'il fut ensuite marchand de vêtements ; et qu'il fonda La Revue socialiste et l'ASIQ après avoir observé le mouvement indépendantiste droitiste de Raymond Barbeau, fondé en 1957. Mais l'on ne sait rien de 
plus précis et, surtout, on ignore tout de la période qui sépare sa naissance en 1914 de son engagement d'après-guerre.

Ce louvoiement apparent entre communisme et nationalisme pique la curiosité de l'historien des idéologies, et suscite des interrogations. Or une étude approfondie des documents relatifs à Roy, et en particulier de ses archives personnelles, révèle un parcours existentiel et idéologique encore plus intéressant. Les hésitations et les tentatives de conciliation de Roy entre nationalisme et socialisme commencent dès sa jeunesse dans les années 1930, décennie au cours de laquelle il est attiré par le fascisme, et se poursuivent jusqu'à la formulation de sa synthèse idéologique au tournant des années 1960. C'est cette évolution de Roy que nous allons retracer ici dans un premier temps, en tentant de déceler, à chaque période, quelle idéologie, du nationalisme ou du socialisme, prime chez ce fondateur de l'indépendantisme socialiste ${ }^{20}$. Nous présenterons ensuite les institutions qu'il a fondées au tournant des années 1960 (La Revue socialiste et l'ASIQ) et leur discours idéologique, avant de conclure sur les raisons pour lesquelles Roy ne fut pas reconnu comme devancier, contrairement à Jacques Ferron, par ceux qui furent en quelque sorte ses héritiers idéologiques, c'est-à-dire les intellectuels de la revue Parti pris et ceux qui, comme eux, firent la promotion d'un nationalisme socialiste de décolonisation pour le Québec (français) ${ }^{21}$.

\section{Le parcours de Roy avant la fondation de La Revue socialiste}

Roy est né en 1914 dans une famille nombreuse de cultivateurs de la Beauce. À l'orée de la Crise, la famille commence à connaître des problèmes économiques, et malgré son grand intérêt pour la lecture et l'histoire, le jeune Raoul se voit interdire de poursuivre ses études ${ }^{22}$. Pendant toutes les 
années 1930, Roy connaît une grande incertitude professionnelle et une grande mobilité géographique. Après ce qui semble être une tentative d'établissement agricole manquée, Roy entre à dix-sept ans chez les Dominicains comme frère convers, même s'il a des doutes sur sa vocation religieuse, probablement en partie pour améliorer son instruction et fuir le manque de ressources culturelles de son milieu ${ }^{23}$. Il en sort au bout d'un an, recommence à travailler l'été sur la terre familiale et commence à travailler comme bûcheron l'hiver, ce qu'il fera pendant le reste des années trente. Il constate dans les chantiers la situation difficile de la jeunesse canadiennefrançaise, ce qui semble toucher un nationalisme déjà enra$\operatorname{ciné}^{24}$.

Mais l'expérience déterminante à l'égard du nationalisme est probablement son séjour à Montréal, où il vient travailler vers 1935, à l'âge de 20 ans. Il semble vivre dans la métropole ce qu'on pourrait appeler un «choc nationaliste». Ne parlant pas l'anglais, il est vivement frappé par le «visage anglais » de la ville, par l'infériorité économique des Canadiens français et par ce qu'il juge être le snobisme ou l'opportunisme anglophile de leur classe commerçante ${ }^{25}$. Ce séjour à Montréal est aussi déterminant pour sa formation intellectuelle : Roy consolide son nationalisme notamment en lisant, à la bibliothèque municipale, " la collection de la revue "l' $A c$ tion nationale" 26 . De retour en Beauce au bout d'un an, il s'abonne au journal nationaliste radical La Nation, de Québec, qui fait à cette époque la promotion de l'indépendance politique du Canada français ${ }^{27}$. Le jeune homme se dira bientôt «nationaliste $\ldots$ xtrèmiste $[$ sic $] \quad \ldots$ et séparatiste ${ }^{28}$ ».

Insatisfait de sa formation intellectuelle et doutant encore de sa vocation agricole, il écrit, de 1937 à 1939, plusieurs lettres à Lionel Groulx pour lui demander conseil et lui exposer ses projets ${ }^{29}$. Dans ces lettres assez confuses, le jeune 
Roy semble tendu entre, d'une part, son goût pour les idées, l'histoire et la défense de la nation et, d'autre part, son désir de fidélité à la vie traditionnelle d'agriculteur, qu'il considère cependant correspondre à une condition diminuée sur les plans intellectuel et matériel, situation qu'il pense être due en grande partie au mépris des gouvernements pour l'agriculture. Cette condition critique des travailleurs du sol et de la nation entière nécessite selon lui un coup de barre radical qui romprait avec l'immobilisme qui profite aux trusts. Et cette prise en main énergique, Roy la conçoit sur l'exemple de l'Italie fasciste et du Portugal corporatiste de Salazar, réputés notamment à cette époque - et surtout dans des journaux comme La Nation - pour leurs réformes agraires ${ }^{30}$.

Mais le fascisme est conçu par Roy comme un moyen radical de libérer le peuple canadien-français du pouvoir des trusts et de la menace que font peser les Anglo-Saxons sur la nation, plutôt que comme un rempart contre le communisme, une doctrine qui semble l'attirer malgré son aspect inquiétant. Un passage de la première lettre de Roy à Groulx condense bien l'état de sa réflexion à l'époque sur le nationalisme et le communisme:

...bien que j'ai $[s i c]$ horreur de la guerre je suis fasciste parce que je crois qu'il nous faut nous les Canadiens bien plus que tout autre peuple une main de fer pour nous sortir de l'ornière où nous sommes tombés.

Je crois qu'il nous faut un chef - un vrai - pour conduire notre peuple de la pauvreté à la richesse matérielle comme intellectuelle et s'il n'était pas possible d'être Fasciste tout en étant catholique eh bien je serais Faciste [sic] quand même

Maintenant si je n'étais pas nationaliste si je n'aimais pas mon pays comme je l'aime et surtout si je croyais qu'il n'est pas possible de vivre dans une patrie Française et. 
que notre peuple est destiné à être anglicisé alors je n'aurais pas de raison d'être Fasciste. en plus si je m'apercevrais $[s i c]$ après tout ce que je viens de dire que le Fascisme n'a pas dompté les financiers qui dominent le monde et que c'est le communisme qui a réussi à faire cela alors je me mettrais à l'étude de cette Doctrine et si elle avait un semblant de logique je l'adopterais

Mais non les communistes dans leur furie de carnages ne se lancent pas sur les gros capitalistes mais sur d'inoffensifs religieux et religieuses ${ }^{31}$ je sais que le Cardinal a presque condamné le corporatisme Fasciste mais ça ne fait rien Je crois que chaque classe de travailleurs étant représentés [sic] par un de ses membres dans un parlement du travail les choses iraient mieux qu'elles vont aujourd'hui Mais en donnant beaucoup de pouvoir aux corporations ne croit-on pas qu'il se produirait des abus sans un homme ayant les pouvoirs nécessaires pour les faire cesser ${ }^{32}$

$\mathrm{Au}$ sujet du communisme, Roy dira encore, dans sa lettre suivante à Groulx :

Il faudra aussi que j'étudie la doctrine communiste. Quest ce [sic] qui s'oppose le plus en moi à ce que je devienne communiste c'est parce que j'aime trop ma patrie pour être internationaliste mais je n'ai rien à dire contre ceux de nos miséreux, de nos exploités, de nos crèves la faim $[s i c]$ de chez nous qui vont au communisme. ils ont au moins ce courage car tout concourent [sic] à faire croire que les capitalistes contrôlent l'église (ce qui n'est pas vrai) et les gouvernants, ce qui est vrai ${ }^{33}$.

Mais le Québec de 1937 - l'année de la fameuse « loi du cadenas »- et la campagne beauceronne sont des milieux assez peu propices à l'étude du communisme, et Roy devra remettre ce projet à plus tard. À la fin des années trente donc, Roy 
adopte le fascisme comme moyen radical d'émancipation des Canadiens français, tout en étant néanmoins intéressé par le communisme.

Pendant la Guerre, Roy quitte la Beauce et s'installe pour de bon à Montréal, où il occupe plusieurs emplois dans le domaine de l'avionnerie jusqu'à la fin du conflit. Selon son témoignage, c'est dans cette ville qu'il découvre un livre du doyen de Canterbury, Hewlett Johnson, qui va transformer profondément sa perception de l'Union soviétique et du socialisme $^{34}$. Il s'agit d'un condensé bilingue du livre La puissance soviétique (1939), qui présente l'URSS sous un jour bien plus favorable que celui où Roy l'avait vue dans la presse québécoise de l'époque ${ }^{35}$. Nous n'avons pu retrouver cette brochure, mais Roy racontera plus tard avoir été frappé notamment par ce qu'on y affirmait sur le traitement favorable par l'URSS des cultures sans écriture : l'URSS de Staline aurait donné à nombre de ces peuples des alphabets qui leur auraient permis de se développer une littérature ${ }^{36}$.

\section{Passage par le mouvement communiste}

Intéressé par cette découverte, Roy se présente au Parti ouvrier-progressiste (nouveau nom du Parti communiste du Canada), qui a publié cette brochure, et demande de l'information. De son entrée dans le P.O.P., Roy donnera plus tard une explication étonnante, qu'il est malheureusement impossible de vérifier : il dira que Danièle Cuisinier, une militante importante de la section québécoise du Parti, lui avait affirmé que celui-ci accorderait une "république francophone » aux Canadiens français, et que c'est cette idée qui l'aurait séduit ${ }^{37}$. Quoi qu'il en soit de cette étonnante affirmation, il est vrai que le P.O.P. fait à cette époque beaucoup d'efforts pour recruter des Canadiens français, ceux-ci étant nettement minoritaires dans les rangs même de la section québécoise du parti. 
Cela l'amène notamment à diluer, dans sa propagande, les éléments de son idéologie qui pourraient facilement déplaire aux Canadiens français, comme l'anticléricalisme, et à jouer sur des thèmes susceptibles de les attirer, comme celui d'une lutte véritable pour la défense de leurs "droits nationaux »: ainsi, l'hebdomadaire Combat, auquel va participer Roy pendant un an, déclarait dans la présentation de son numéro initial, le 23 novembre 1946 :

COMBAT réclame le respect des droits nationaux, politiques et économiques des Canadiens français. Nous nous opposons à un Louis St-Laurent lorsqu'il prétend que le parlement du Canada légalement peut abolir l'usage du français comme langue officielle. Au chapitre de l'autonomie provinciale, nous ne nous arrêtons pas à la doctrine stérile et, au fond, anti-nationale, d'un Maurice Duplessis. Nous réclamons des mesures pour freiner le pouvoir des trusts, pour réaliser l'égalité nationale, - économique, politique et sociale des Canadiens français ${ }^{38}$.

Sur le plan religieux, l'hebdomadaire évite l'anticléricalisme et rapporte fréquemment les propos d'ecclésiastiques européens favorables à une coopération avec les socialistes, ou encore dément l'image persécutrice des régimes socialistes ${ }^{39}$.

Entré au Parti ouvrier-progressiste, Roy participe à ce journal dès sa fondation, et il obtient rapidement par le biais du Parti un emploi de "rédacteur français " (traducteur) du journal Searchlight, de l'Union des marins canadiens, qui sera son gagne-pain pendant plus de deux ans ${ }^{40}$. Mais c'est dans Combat que Roy signe la plupart de ses articles de l'époque, c'est-à-dire quelques articles de reportage, des notes de voyage en Europe et une enquête sur la jeunesse ${ }^{41}$.

Cette entrée au P.O.P. donne donc pour la première fois à Roy la possibilité de gagner sa vie en écrivant, une conquête 
pour ce jeune intellectuel largement autodidacte. Mais sur le plan idéologique, il faut se demander si cela exige de lui une adhésion complète à l'internationalisme socialiste et un abandon complet de ses préoccupations nationalistes canadiennes-françaises. Il nous semble que non, et ce pour plusieurs raisons. Premièrement, parce qu'il semble qu'à cette époque, comme durant une bonne partie de son histoire, le Parti adhère à l'idée que les Canadiens français constituent un groupe particulièrement exploitét2. Par conséquent, le Parti, par le biais de son journal Combat, critique ce qu'il juge être «l'hypocrisie » de l'autonomisme de Duplessis en soulignant l'abaissement de celui-ci (et des autres pouvoirs politiques canadiens) devant les trusts et les financiers de Wall Street. À un niveau plus symbolique, le journal critique l'histoire nationaliste de Lionel Groulx, mais il le fait en appelant à une revalorisation "des véritables traditions démocratiques et révolutionnaires des Canadiens français » et de la mémoire du mouvement des Patriotes de 1837-1838 (un thème qui sera d'ailleurs repris, dix ans plus tard, par l'ASIQ et La Revue socialiste qu'animera Roy ${ }^{43}$. Deuxièmement, l'adhésion au Parti communiste canadien ne nécessite pas un reniement complet du nationalisme parce que le socialisme canadien est lui-même animé par des préoccupations nationales : la libération des travailleurs canadiens passe par la socialisation de l'économie canadienne, qui est conçue en partie comme une résistance à l'impérialisme américain ${ }^{44}$. Ainsi, pour ne donner que des exemples rapprochés de Roy, l'Union des marins canadiens lutte, à l'époque où celui-ci y travaille, pour la préservation d'une marine marchande canadienne et pour les droits des marins canadiens dans l'après-guerre ${ }^{45}$.

À cet égard, il paraît significatif que la principale série de reportages à laquelle participe Roy dans Combat a pour sujet une affaire d'importation de main-d'œuvre étrangère à bon 
marché dans la Beauce, sa région natale. Ludger Dionne, un industriel de Saint-Georges de Beauce siégeant à la Chambre des communes, avait fait venir d'Allemagne une centaine de jeunes Polonaises qui s'engageaient à travailler à faible salaire pour deux ans dans ses usines de rayonne et qui étaient obligées de demeurer en pension dans un couvent ${ }^{46}$. Le journal présente cette manœuvre comme de l'esclavage et comme une menace pour la classe ouvrière canadienne, parce qu'elle exerce une pression à la baisse sur les salaires. Ici, socialisme et nationalisme se conjuguent autour de la question de l'immigration, une thématique qui sera très présente dans $L a R e$ vue socialiste que fondera Raoul Roy douze ans plus tard.

Raoul Roy pouvait donc dans les années 1946-1949, adhérer au Parti communiste canadien sans renier complètement son nationalisme ${ }^{47}$. Néanmoins, durant cette période d'engagement socialiste, des éléments idéologiques nouveaux se mettent en place qui feront partie de sa doctrine au début des années 1960. Il y a d'abord, évidemment, l'adoption du socialisme comme moyen radical et puissant d'émancipation des travailleurs exploités par les puissances industrielles et financières qui contrôlent selon lui les gouvernements; on pourrait dire que le socialisme prend en cela la place qu'occupait le fascisme dans la pensée de Roy à la fin des années 1930. Deuxièmement, c'est probablement à cette époque que Roy acquiert une méfiance vis-à-vis de ce qu'on appelle le " nationalisme traditionnel », dont sait bien se servir Duplessis et qui lui semble trop frileux et rétrograde pour susciter des changements importants; Roy refusera ainsi, dans les années 1960, d'associer le mot de «nationalisme » à sa doctrine, qu'il préférera appeler « socialisme décolonisateur $»^{48}$. Troisièmement, c'est probablement aussi pendant cette période qu'il développe, soit par un voyage au Portugal, soit par la lecture, une méfiance profonde vis-à-vis du corporatisme 
en tant que doctrine sociale juste et efficace. Le journal Combat publiait alors par exemple sur le Portugal de Salazar, un modèle pour plusieurs réformateurs nationalistes, une série d'articles intitulée "Derrière le rideau de fer portugais : Salazar, pantin fasciste ${ }^{\text {t) }}$ ». Ce rejet du corporatisme est important, puisqu'il sera un des éléments qui pousseront Roy à fonder l'indépendantisme de gauche en 1959, alors que la doctrine sociale de l'Église et le corporatisme inspiraient le mouvement indépendantiste de Barbeau.

Deux événements vont amener Roy à quitter le mouvement communiste. D'abord, à l'automne 1947, une crise secoue la section québécoise du Parti ouvrier-progressiste. Des militants canadiens-français importants sont accusés de "déviation nationaliste » et ils quittent le Parti, ce qui entraîne le départ d'une grande partie des membres canadiens-français du Parti ${ }^{50}$. Roy affirme avoir quitté le Parti à cette époque ${ }^{51}$. Puis, en 1949, l'Union des marins canadiens traverse une grave crise à cause de son affiliation communiste, et Roy perd son emploi ${ }^{52}$.

Il cesse alors de publier pour une dizaine d'années. Pendant les années cinquante, il ouvre à Montréal des boutiques de mercerie. Il fait d'assez bonnes affaires pendant un certain temps, mais le commerce semble décliner à la fin des années 1950, pour diverses raisons. Pendant ces années, il côtoie quotidiennement des Juifs, très nombreux dans l'industrie du vêtement, et il semble concevoir alors une frustration face à certaines de leurs attitudes (commerciales ou autres), quoiqu'il soit ensuite demeuré assez discret à ce sujet, surtout dans les années $1960^{53}$. Il y trouve probablement une autre confirmation de l'importance fondamentale de la question nationale dans les problèmes des Canadiens français, une conviction qui transparaîtra clairement dans ses écrits ultérieurs. 


\section{La fondation d'un indépendantisme de gauche}

C'est la renaissance du mouvement d'indépendance du Québec en 1957, sous l'impulsion de Raymond Barbeau, de sa revue Laurentie et de son groupe l'Alliance laurentienne, qui incita Roy à se relancer dans l'activité militante après presque dix ans de relative inactivité. Ayant découvert par hasard la revue Laurentie au début de 1957, il affirme avoir été enchanté de découvrir la renaissance de l'idée d'indépendance, tout en étant rebuté par le caractère droitiste de l'idéologie promue par le mouvement de Barbeau. L'Alliance laurentienne faisait en effet la promotion de l'indépendance du Québec en l'assortissant d'un projet d'État catholique et corporatiste conforme à la doctrine sociale de l'Église, le régime de référence étant le Portugal de Salazar; en outre, elle mettait aussi l'accent sur le rôle des élites dans la réalisation de l'indépendance ${ }^{5+}$. Laurentie et l'Alliance (toutes deux fondées en janvier 1957) étant les seules institutions à faire la promotion de l'indépendance du Québec, ce projet risquait selon Roy d'être associé fortement et durablement à la droite, ce qui était inacceptable à ses yeux tant pour des raisons intrinsèques de sensibilité idéologique que pour des raisons stratégiques ayant trait à la promotion de cette option politique ${ }^{55}$.

Se considérant homme de gauche et intéressé par le socialisme, Roy rejetait en soi le corporatisme, l'idéal d'État chrétien et l'élitisme de l'Alliance. Mais il jugeait également dangereuse cette association de l'indépendantisme à la droite parce qu'elle donnait à cette option politique un visage « réactionnaire » qui risquait selon lui de la discréditer durablement dans un contexte où l'air du temps était libéral et où l'intelligentsia occidentale, rejetant vivement les valeurs et les modèles de droite, adhérait à un progressisme de gauche plus proche du socialisme $^{56}$. Pour Roy et ses futurs collaborateurs, Barbeau, en campant le séparatisme à droite, risquait de tout gâcher ${ }^{57}$. 
Rejetant le droitisme et le corporatisme de l'Alliance et persuadé qu'ils menacent de nuire à la cause de l'indépendance, Roy est convaincu de la nécessité de donner un visage de gauche au séparatisme. C'est pour cela qu'il fonde en 1959 La Revue socialiste (1959-1965), deuxième revue indépendantiste d'après-guerre et la première à conjuguer au Québec les idées d'indépendance et de socialisme. Cette revue sera pendant environ quatre ans la seule à faire la promotion de l'indépendantisme socialiste, jusqu'à la fondation de Parti pris (et, dans une moindre mesure, du journal felquiste La Cognée) en octobre 1963. Elle marque le début du courant qu'on peut appeler "socialisme et indépendance ", et qui connaitra un grand succès au sein de la gauche québécoise au cours des années 1960 et 1970. Par ailleurs, Roy participe au même moment à la mise sur pied de l'éphémère Ligue de la maind'œuvre native du Québec, qui conjugue nationalisme et socialisme sur un mode populiste dans son combat pour le contrôle et l'arrêt de l'immigration dans la province, une préoccupation qui demeurera centrale dans le discours de Roy jusqu'à la fin de sa vie ${ }^{58}$.

C'est l'été suivant (août 1960) que Roy fonde, dans la foulée de la Société des amis de La Revue socialiste (1959), l'Action socialiste pour l'indépendance du Québec (ASIQ), deuxième groupe depuis la guerre à être voué à la promotion de l'indépendance du Québec. Ce petit groupe - il ne comptera probablement jamais plus que quelques dizaines de membres - fait la promotion des idées de la revue en organisant des manifestations ainsi que des assemblées publiques (conférences et débats) permettant la discussion, au sein de la gauche québécoise, de l'idée d'indépendance. Il contribue aussi à la diffusion du socialisme et de l'idéologie de la décolonisation par le biais d'un service de librairie que Roy met sur pied à cette époque. L'ASIQ sera active jusque vers 1963, date à 
laquelle Roy doit s'exiler pour quelques mois en France parce qu'il est soupçonné d'être un meneur de la première vague du Front de libération du Québec (FLQ), dont plusieurs membres ont fréquenté son mouvement. Ce séjour dans l'Hexagone confortera encore la conviction qu'avait Roy de la nécessité d'associer clairement l'indépendantisme québécois à la lutte de la gauche pour la décolonisation des peuples du Tiers-monde; conviction qui amènera Roy, à son retour, à participer au journal populiste du Front républicain pour l'indépendance (Québec libre) et à lutter pour faire du Nouveau Parti ${ }^{59}$ une formation indépendantiste.

Les institutions qu'a fondées et dirigées Roy (La Revue socialiste et l'ASIQ surtout) connurent un faible succès numérique, si l'on compare le tirage de La Revue socialiste à celui de Laurentie ou encore la taille de l'ASIQ à celle de l'Alliance laurentienne ou du Rassemblement pour l'indépendance nationale $(\mathrm{RIN})^{61}$. Elles eurent cependant une visibilité médiatique appréciable et surtout, elles furent le lieu de l'élaboration et de la première diffusion d'un discours qui, relayé par des organes plus puissants comme la revue Parti pris et repris par la jeune génération de militants nationalistes de gauche, allait connaitre beaucoup de succès dans les années suivantes et donner à la fois un nouveau visage et un nouvel essor au nationalisme canadien-français (bientôt " québécois ») ${ }^{61}$.

Ce discours, qui fusionne nationalisme et socialisme autour de l'idée de décolonisation, s'infiltre dans le débat qui fait alors rage au Canada français entre les nationalistes de droite ou non séparatistes et la gauche antinationaliste, pour renvoyer les deux camps dos à dos. Il accuse l'une et l'autre tendances d'avoir trahi (et de continuer à trahir) le peuple canadien-français, au double sens de classes populaires et de nation. Il reproche aux nationalistes leur passéisme, leur conservatisme social, leur compromission avec le gouvernement 
Duplessis et, partant, avec ceux qui exploitent les masses canadiennes-françaises, ainsi que l'ineptie de leur doctrine sociale (en particulier le corporatisme). Il fustige aussi, évidemment, leur rejet de l'indépendantisme. Pour éviter de leur être associé, il refuse le plus souvent qu'on qualifie son mouvement de $"$ nationaliste $»^{62}$.

Mais Roy fustige avec autant sinon plus d'ardeur l'antinationalisme et les positions centralisatrices de la gauche socialiste et libérale canadienne-française et canadienne, qu'il accuse de contribuer à l'aliénation croissante du peuple canadien-français et à son éventuelle disparition. Un régime socialiste centré à Ottawa ne pourrait selon lui signifier que l'effritement du pouvoir des Canadiens français sur leur propre destinée et une perpétuation, sous une forme nouvelle et accrue, de la domination des Anglo-Canadiens sur les Canadiens français. Sous prétexte de vouloir déprolétariser les $\mathrm{Ca}$ nadiens français, les socialistes ne réussiraient ainsi qu'à les dépouiller de cette dimension humaine fondamentale qu'est l'identité nationale. La dénonciation des socialistes aboutit à une critique du socialisme orthodoxe au nom d'un socialisme plus authentique et de la recherche d'une pensée de gauche véritablement appropriée aux besoins des Canadiens français, non seulement prolétarisés mais colonisés. Roy trouvera des éléments d'une telle pensée chez les penseurs francophones de la décolonisation (Frantz Fanon, Albert Memmi, Jacques Berque), eux aussi critiques des vieilles gauches suspectes de complicités impérialistes.

Cette colonisation du Canada français qui légitime l'indépendantisme, Roy et ses collaborateurs de La Revue socialiste s'emploient à en illustrer les diverses manifestations économiques, démographiques, politiques, culturelles et linguistiques. Ils élaborent ainsi, dans le contexte du débat alors en cours au sujet des causes et des responsabilités de la pau- 
vreté et du « retard » du Canada français, l'une des positions les plus radicalement nationalistes au sujet de l'histoire et de la situation des Canadiens français. La profondeur de l'aliénatíon des Canadiens français, véritable classe ethnique au sein du Canada, entraîne la nécessité d'une conjonction de solutions radicales : l'indépendance et le socialisme, que Roy présente sans cesse comme mutuellement nécessaires, dans le but polémique à la fois d'infléchir le séparatisme vers la gauche et de rendre indépendantiste la gauche canadienne-française.

L'analyse de cet amalgame idéologique révèle cependant une nette primauté du nationalisme dans le discours de Roy, pour qui la révolution la plus importante demeure toujours l'indépendance. Le socialisme est surtout conçu comme un outil permettant de réaliser la libération économique des Canadiens français et de leur assurer une véritable indépendance. En plus de certaines mesures de sécurité sociale, il consisterait surtout en la nationalisation (ou "socialisation») par l'État québécois d'entreprises et de secteurs économiques contrôlés par des intérêts étrangers, anglo-canadiens ou américains en particulier. C'est un socialisme « mitigé » qui ne tend pas à l'abolition de la propriété privée ni au renversement du rapport de classes au sein du Canada français, dont Roy minimise d'ailleurs l'importance.

Le nationalisme de Roy, au contraire, est à la fois radical et intégral, ce que légitime l'idéologie de la décolonisation dont le militant se fait le propagateur. La libération nationale a préséance sur le respect de certains droits formels de la « minorité colonialiste » du Québec, et une certaine violence (surtout à l'endroit des symboles du « colonialisme anglo-saxon ») apparait parfois nécessaire ${ }^{6.3}$. Si c'est dans le peuple que Roy voit le plus souvent le salut de la nation, il envisage généralement sans trop de malaise une alliance de classes « anticolo- 
nialistes » en vue de l'indépendance; à maintes reprises, d'ailleurs, Roy démontre qu'il est prêt à collaborer avec des groupes indépendantistes d'autres couleurs idéologiques en vue de faire avancer la cause de l'indépendance. Le nationalisme de Roy s'exprime aussi dans sa valorisation d'une grande partie de l'héritage culturel canadien-français (qui contribue à l'éloigner de l'anticléricalisme) ainsi que de la spécificité culturelle de ce peuple, une motivation fondamentale de l'indépendantisme du militant : l'indépendance doit empêcher l'étouffement et permettre l'épanouissement d'une culture originale, latine, qui tranche avec celle de l'Amérique du Nord anglo-saxonne ${ }^{64}$.

Ce nationalisme culturel est le versant positif de la méfiance profonde de Roy envers les anglophones du Québec, du Canada et d'ailleurs. On a peine à trouver mention, dans la revue, d'un anglophone qui, consciemment ou inconsciemment, ouvertement ou secrètement, ne méprise pas les Canadiens français et ne désire pas leur perte; au mieux, le progressiste anglo-saxon est incapable de comprendre la nécessité d'une protection de la culture canadienne-française $e^{65}$. La Revue a par ailleurs tendance à occulter la diversité des situations socio-économiques des Anglo-Québécois, en assimilant trop souvent anglais et bourgeois; ce qu'elle justifie en affirmant que le prolétariat anglophone a tendance à être solidaire d'abord de son groupe linguistique, avant d'être solidaire de sa classe, parce qu'il se sait privilégié par les employeurs et les institutions anglophones. La Revue socialiste se défendra bien de faire du racisme quand le RIN déclarera, peut-être pour se distinguer de celle-ci : «Nous n'en voulons pas aux Anglais ». Pour la Revue, tous les anglophones du Québec profitent objectivement de la colonisation du Québec; et ceux qui n'appuient pas l'effort de libération nationale des Canadiens français en sont les complices, quoique à 
des degrés variables qu'il faut avoir la justice de marquer. Il n'est en général pas question de mettre tous les anglophones du Québec à la porte, mais seulement les grands colonialistes $^{66}$.

Envers les autres minorités ethniques et l'immigration, l'attitude de Roy et de sa revue est surtout faite de méfiance justifiée par un argumentaire qui tient à la fois du nationalisme et du socialisme, mais surtout du premier. Roy qualifie de "colonisation démographique » l'usage que fait selon lui de l'immigration la «bourgeoisie colonialiste » anglophone. En faisant venir au Québec un grand nombre d'immigrants anglophones alors même que le taux de chômage est alarmant, le patronat ou "colonat » anglophone cherche selon lui à la fois à faire baisser les salaires, à désamorcer les revendications ouvrières (par l'emploi de briseurs de grève étrangers) ainsi qu'à diluer graduellement le poids démographique des Canadiens français, peuple prolétaire, dans le but de réduire leur pouvoir politique ainsi que les possibilités de leur survivance culturelle ${ }^{67}$. En cela, cette stratégie s'inscrirait selon Roy dans la longue durée de la colonisation britannique, qui aurait toujours souhaité et projeté l'assimilation des Canadiens. Les immigrants, quant à eux, sont accusés de jouer le rôle de "minorités satellites " et d'instruments du pouvoir anglo-saxon, adoptant sa langue et s'adossant à lui pour profiter de ses faveurs, niant à la fois leur devoir de respect envers les Canadiens français sur leur territoire national et le principe de la solidarité internationale des prolétaires et menaçant, en dernière analyse, la position des Canadiens français au Québec et au Canada. Néanmoins, à la méfiance et au ressentiment nationalistes se mêle un désir, nationaliste lui aussi, d'intégrer ces groupes à la nation francophone par diverses mesures allant de l'incitation (sécularisation du Canada français, mise sur pied d'un réseau scolaire non confession- 
nel) à la coercition. En somme, au tournant des années 1960, le nationalisme prime nettement (et fortement) le socialisme dans cette première version du nationalisme socialiste québécois qu'élaborent Roy et ses collaborateurs.

\section{Un pionnier marginal}

Roy fut donc l'un des pionniers de l'indépendantisme socialiste au Québec, mais il eut peu de disciples et de reconnaissance alors même que l'idéologie de la décolonisation du Québec par l'indépendance et le socialisme prenait un essor important dans les années 1960, notamment après le lancement de Parti pris en octobre $1963^{68}$. Il convient de se pencher un moment sur les causes possibles de ce fait de prime abord un peu surprenant.

Certaines d'entre elles tiennent vraisemblablement à la personnalité, aux attitudes, voire à la volonté de Roy. Jacques Ferron affirmait avoir su dès le départ qu'avec « [Roy] comme chef [de l'ASIQ], nous n'y serions jamais nombreux ${ }^{69} \%$. Yves Ménard, qui milita aux côtés de Roy dans les années 1980, écrit quant à lui que Roy n'avait pas l'âme d'un grand organisateur $^{70}$, et il est probable que le militant ait préféré les petits groupes et que la marginalité ne l'ait pas trop dérangé. Roy ne semble pas non plus avoir eu le talent oratoire et le charisme de rassembleurs comme Raymond Barbeau ou Pierre Bourgault. En outre, intellectuel autodidacte et original sans formation classique ou marxiste "scientifique ", il était mal outillé pour impressionner de jeunes intellectuels qui, formés au collège classique ou à l'université, recherchaient des maîtres à penser sérieux et prestigieux : ses idées originales, ses néologismes souvent étranges - voire farfelus - et son populisme agressif ont pu les rebuter. Le dogmatisme et le dirigisme dont Roy faisait preuve dans la discussion et au sein de ses mouvements ne pouvaient que les inciter davantage à fon- 
der leur propre mouvement au lieu de militer à l'ASIQ ou d'écrire dans La Revue socialiste. Enfin, l'âge moyen sans doute plutôt élevé des membres du mouvement de Roy n'aidait probablement pas à y attirer de jeunes intellectuels ${ }^{71}$.

Il semble d'ailleurs qu'à part André Major, les fondateurs de Parti pris se méfiaient de Roy ${ }^{72}$. Cela pouvait provenir des facteurs qu'on vient d'énumérer, mais cela renvoyait certainement aussi à des divergences de valeurs, de sensibilité et d'idéologie. Les jeunes idéologues croyaient probablement subodorer un certain racisme sous l'ardente opposition de Roy à l'immigration et sa critique des comportements des minorités ethniques. Ils étaient également bien plus anticléricaux que lui, et méprisaient probablement sa relative prudence à l'endroit de l'Église ainsi que sa conception de l'importance majeure du catholicisme dans la culture de la nation canadiennefrançaise $^{73}$. On pourrait d'ailleurs avancer que Roy et ces jeunes idéologues avaient des perceptions assez différentes de la culture canadienne-française - et de la culture populaire en particulier -, le diagnostic des plus jeunes étant souvent plus pessimiste que celui de leur devancier; d'où leur insistance apparemment plus grande sur la nécessité d'une révolution culturelle profonde. Par ailleurs, sur le plan théorique, un bref survol de la revue Parti pris semble révéler que ses jeunes rédacteurs plaçaient bien plus d'espoirs que Roy dans le marxisme en tant qu'outil d'analyse et de transformation de la société, même si plusieurs observateurs ont jugé par la suite que le marxisme de la revue était superficiel ${ }^{74}$.

Enfin, dans ce domaine plus difficile à évaluer qu'est celui des sensibilités, un fossé séparait vraisemblablement ces jeunes intellectuels de Roy, qui avait déjà quarante-neuf ans lors de la fondation de Parti pris en 1963. Au niveau des valeurs morales d'abord, Roy était probablement plus conservateur que ses jeunes successeurs. Le qualificatif de «béné- 
dictin » dont Ferron l'affuble à un endroit renvoie probablement non seulement à ses habitudes de travail, mais aussi à son mode de vie sobre et à sa personnalité peut-être un peu sèche ${ }^{75}$. Roy n'affichera jamais que du dédain pour le libéralisme moral des tenants de la contre-culture, hippies et autres. En outre, Roy était avant tout un militant nationaliste passionné d'actualité et de politique ; il ne partageait pas l'intérêt des jeunes écrivains de Parti pris pour les arts et la littérature, ni, peut-être, leur conviction que ces dimensions de l'existence étaient intimement liées à la politique ${ }^{76}$.

On voit donc que même si les jeunes indépendantistes socialistes de Parti pris partageaient une bonne part de l'idéologie de Roy, notamment le souci primordial de faire l'indépendance et la volonté de concevoir un "socialisme québécois " adapté à la réalité "coloniale » du Québec, bien des choses les séparaient du fondateur de La Revue socialiste et permettent d'expliquer leur décision de fonder leurs propres institutions de même que celle de passer sous silence cette filiation intellectuelle qu'ils jugeaient peut-être honteuse.

À partir de 1963 donc, année où furent fondés non seulement la revue Parti pris, mais aussi le Parti socialiste du Québec et le FLQ, les courants du socialisme québécois et de l'indépendantisme socialiste s'enrichirent de nouvelles voix et prirent un essor nouveau. Roy le précurseur vit le composé idéologique qu'il avait élaboré repris par d'autres, mieux diffusé et souvent substantiellement transformé. Cette multiplication des variantes de l'idée de "socialisme et indépendance " l'amena dans les années qui suivirent à préciser davantage le sens de son "socialisme décolonisateur» ainsi que les objectifs et les moyens légitimes de l'indépendantisme socialiste, et à réagir contre les interprétations "hérétiques » d'autres intellectuels. 
Le long dialogue qui concluait le dernier numéro de $L a$ Revue socialiste participait déjà de cet effort de clarification ${ }^{77}$. Le texte, qui faisait dialoguer trois personnages incarnant respectivement les positions du "nationalisme traditionnel », du "pseudo-marxisme » fédéraliste et du "socialisme anticolonialiste ", réaffirmait la primauté essentielle et la priorité des objectifs nationaux ainsi que leur coïncidence à peu près parfaite avec les objectifs de libération sociale. Cependant Roy n'y critiquait encore personne ouvertement, même s'il semble que ce texte ait été suscité en partie par l'apparition de la revue Révolution québécoise, de Pierre Vallières et Charles Gagnon, qui refusait d'accorder à l'indépendance la priorité (même chronologique) sur la lutte sociale ${ }^{78}$.

Dès l'année suivante, les critiques de Roy deviennent plus franches et directes. Dans le second numéro de son bulletin L'indépendantiste (juin 1966), Roy adresse ses premières critiques et toute une série de questions à Parti pris ${ }^{79}$. Il se montre aussi très sévère à l'égard de Pierre Vallières, avec qui des membres de cette revue avaient fondé l'année précédente le Mouvement de libération populaire, remettant en question la priorité (temporelle) qu'ils avaient jusque-là accordée à l'indépendance et reniant leur position d'appui tactique à la bourgeoisie (en l'occurrence le RIN) ${ }^{80}$. Vallières considérait que les deux révolutions (nationale et sociale) devaient se faire simultanément et il subordonnait l'indépendance à l'objectif socialiste : si celle-là ne servait pas clairement celui-ci, elle ne méritait pas d'être faite. Le groupe felquiste qu'il animait n'avait d'ailleurs pas craint d'attaquer des entreprises canadiennes-françaises, ce qui aux yeux de Roy était si nuisible pour l'image de l'indépendantisme qu'on pouvait parler de «sabotage $»^{81}$.

En outre, Vallières concevait la double révolution québécoise, socialiste et nationale, comme faisant partie intégrante 
d'une révolution mondiale dirigée contre l'impérialisme américain. Roy jugera très dangereuse, pour un petit peuple encore fragile, l'idée de se lancer dans ce qu'il appelle la "guérilla de la révolution planétaire " contre le géant américain. Qualifiant cette tendance de "mystique » et "d'anarchiste», il fustigera ces « internationaleux » qui veulent selon lui lancer les Canadiens français dans une "grande tuerie $»^{82}$. Roy exhortera Parti pris et les socialistes indépendantistes à restreindre au minimum leur anti-américanisme, pour éviter d'éveiller l'hostilité de la superpuissance à l'endroit du mouvement indépendantiste : la lutte prioritaire devait à tout prix demeurer celle contre le pouvoir d'Ottawa, qui seule avait des chances de fonctionner ${ }^{83}$.

Parti pris ne répondit pas directement aux critiques et aux questions que lui adressait Roy par le biais de son obscur bulletin $^{8+}$. Roy pouvait en outre se plaindre de ce qu'un rédacteur de la revue avait repris son terme de "socialisme décolonisateur » en lui donnant un sens très différent ${ }^{85}$. Il en vint même à concevoir quelques doutes sur la pertinence ou la prudence d'employer le terme "socialisme » pour désigner sa doctrine de libération collective : s'il ne regrettait pas d'avoir contribué au « dédouanement » du socialisme au Canada français, n'avait-il pas cependant, en choisissant ce mot dix ans plus tôt, semé lui-même le germe de cette "confusion » qui régnait maintenant selon lui dans la gauche nationaliste au sujet de la nature et de la priorité des luttes à faire ${ }^{86}$ ? On ne s'étonnera donc pas de voir plus tard Roy délaisser sans cérémonie le terme de «socialisme » pour employer plutôt ceux de «décolonisation » et d'« anticolonialisme ».

Les années 1970 seront pour Roy l'occasion d'une autre réaffirmation de ses positions sur le socialisme à cause de la montée des mouvements marxistes-léninistes, qui s'opposeront de plus en plus à l'indépendantisme à mesure que s'ạp- 
prochera du pouvoir le Parti québécois, qui avait rallié la majorité des socialistes indépendantistes. Ainsi, en 1976, Roy réédite sous le titre Socialisme! Lequel? le dialogue paru en 1965 dans le dernier numéro de La Revue socialiste, qui illustre l'opposition entre son "socialisme décolonisateur» et le socialisme marxiste traditionnel ${ }^{87}$. Puis, l'année suivante, il revient à la charge en publiant l'opuscule Marxisme: mépris des peuples colonisés?, dans lequel il expose ce qu'il estime être "l'impérialisme » de Marx et Engels à l'endroit des peuples jugés réfractaires à la marche du Progrès, ainsi que l'oppression que firent subir les régimes soviétiques aux nationalité $^{88}$. Dénonçant ce qu'il juge être le «mysticisme » et la «trahison » consciente ou inconsciente des intérêts du peuple canadien-français par les mouvements marxistes-léninistes et les syndicats qui s'opposent au Parti québécois au pouvoir, Raoul Roy s'emploie donc, à la fin des années 1970, à dénoncer et à tenter de convertir ces socialistes qui, au nom des intérêts de la classe ouvrière, risquent selon lui de faire manquer au peuple canadien-français sa véritable libération, la libération nationale.

Bien entendu, cette étude des rapports entre nationalisme et socialisme dans la pensée de Raoul Roy ne peut constituer que le commencement d'un examen plus approfondi de la question des relations entre ces deux idéologies au sein du socialisme et du nationalisme de gauche au Québec dans les années 1960. Le discours de Roy n'est qu'une position extrême d'un débat qui agitera ces mouvances durant cette décennie.

À bien des égards, la pensée de Roy fait aussi figure de transition entre deux visages du nationalisme francophone ${ }^{89}$. Tout en préfigurant par plusieurs aspects le nationalisme québécois de gauche qui prend son essor au cours des années 1960, elle conserve de nombreuses traces de la formation de 
Roy au sein du nationalisme canadien-français groulxiste et radical des années $1930^{\circ 0}$. Roy parle le langage nouveau de l'indépendance, du socialisme et de la décolonisation, mais sa définition de la nation demeure canadienne-française plutôt que québécoise et sociologique (voire ethnique) plutôt que civique ou territoriale. Sa méfiance et son ressentiment déclarés vis-à-vis de l'immigration et des communautés ethniques qu'il juge hostiles aux Canadiens français le rapprochent également davantage de la tradition nationaliste que du nouveau nationalisme soucieux de montrer au monde un visage tolérant et ouvert. En outre, son laïcisme modéré et sa prudence vis-à-vis de l'Église, qu'il juge faire partie de l'identité canadienne-française, tranchent avec l'anticléricalisme et le laïcisme ardent de bien des néo-nationalistes socialistes ${ }^{91}$. Cette position médiane et cette hybridité de l'idéologie de Roy la condamnaient probablement à la marginalité à une époque où le nouveau nationalisme québécois de gauche, jamais à court de détracteurs, luttait pour se distinguer de ses gênants ancêtres droitistes.

\section{NOTES}

${ }^{1}$ Nous remercions les professeurs Roberto Perin et Pierre Trépanier, ainsi que M. Patrick Dionne, d'avoir bien voulu lire et commenter ce texte, de même que les évaluateurs et l'équipe de la revue Mens pour leur correction attentive et leurs judicieuses suggestions. Bien entendu, nous gardons l'entière responsabilité des erreurs qui pourraient y subsister. La rédaction de cet article a été rendue possible par le soutien financier du Conseil de recherches en sciences humaines du Canada (CRSH).

${ }^{2} \mathrm{Il}$ y a aussi une forme de prudence dans cette façon de farre : en s'en tenant aux explications globales et impersonnelles, on peut risquer de se tromper sans craindre d'être trop injuste envers ces personnes que sont les acteurs historiques, ici conçus davantage comme l'expression de forces sociales que comme des individus situés historiquement et faisant des choix dans un contexte social, économique, politique et culturel donné. 
${ }^{3}$ Dale C. Thomson, Jean Lesage e The Quiet Revolution, Toronto, Macmillan, 1984,500 p.

${ }^{+}$Kenneth McRoberts, Queber: Social Change and Political Crisis. $3^{\text {nd }}$ edition with a Postscript, Toronto, McClelland \& Stewart, 1993, 556 p.

${ }^{5}$ William D. Coleman, The Independence Movement in Quebec, 1945-1980, Toronto, University of Toronto Press, 1984, 274 p.

"Cela est particulièrement étonnant dans le cas de Coleman, vu le titre de son livre.

${ }^{7}$ Léon Dion, La révolution déroutée, 1960-1976, Montréal, Boréal, 1998, 324 p.

${ }^{8}$ Les ouvrages classiques des historiens Maurice Séguin et Jean-Claude Robert, bien que très différents par leur nature, ont en commun l'ambition d'expliquer les origines historiques de l'indépendantisme et de situer sa genèse dans la longue durée de l'histoire québécoise ; ils ne donnent par conséquent que peu de détails sur le mouvement indépendantiste relancé en 1957 : Maurice Séguin, L'idée d’indépendance au Québec. Genèse et historique, Trois-Rivières, Boréal express, 1968, 67 p. ; Jean-Claude Robert, $D u$ Canada francais au Québec libre : histoire d'un mouvement indépendantiste, Paris, Flammarion, 1975, 323 p. Cela est encore plus vrai de l'étude de Fernand Ouellet, «Les fondements historiques de l'option séparatiste dans le Québec », Canadian Historical Review, XLIII, n" 3 (septembre 1962), p. 27-42.

'Pierre Godin, René Lévesque, Montréal, Boréal, 1994, 4 v. ; idem, René Lévesque, un homme et son rêve, 1922-1987, Montréal, Boréal, 2007, 716 p. ; il faut mentionner également la série d'ouvrages du même auteur sur la Révolution tranquille, en particulier son livre Les frères divorcés, Montréal, Éditions de l'Homme, $1986,360 \mathrm{p}$. Autre figure marquante du mouvement indépendantiste, le tribun Pierre Bourgault a récemment fait l'objet d'une riche biographie : Jean-François Nadeau, Bourgault, Montréal, Lux, 2007, 606 p. Pour être juste, il faut dire que ces grandes biographies portraiturent souvent des militants pionniers et des personnages dits « secondaires ", mais cela est souvent fait rapidement, parfois négligemment et en général sans référence adéquate aux sources. En outre, ces portraits sont souvent biaisés par la perspective du biographe ou encore celle du personnage qu'il étudie et de son entourage.

1" Réjean Pelletier, Les militants du R.I.N., Ottawa, Éditions de l'Université d'Ottawa, 1974, 82 p. ; André d'Allemagne, Le R.I.N. de 1960 à 1963 : étude d'un groupe de pression au Québec, Montréal, Éditions l'Étincelle, 1974, 160 p. 
"Témoin le documentaire de Jean-Claude Labrecque, Le RIN, qui ne mentionne qu'au passage l'Alliance laurentienne, dont ont pourtant fait partie les deux principaux fondateurs du Rassemblement (Marcel Chaput et André d'Allemagne), et qui tend à faire de d'Allemagne non seulement le penseur du mouvement, mais aussi, en quelque sorte, le père de l'indépendantisme. Ainsi le film s'ouvre-t-il sur l'épisode de la conversion par d'Allemagne de Pierre Bourgault, qui devint rapidement l'un des plus ardents (et des plus efficaces) chantres de l'idée de l'indépendance du Québec. Jean-Claude Labrecque, Le RIN, Montréal, Productions Virage, 2002, couleur, 78 min.

${ }^{12}$ Une exception notable est le substantiel mémoire de maitrise d'Éric Bouchard sur Raymond Barbeau et l'Alliance laurentienne : Éric Bouchard, Raymond Barbeau et l'Alliance laurentienne : les ultras de l'indépendantisme québécois, Mémoire de maîtrise (histoire), Université de Montréal, 1997, 219 p. JeanMarc Brunet, ami de Barbeau, a également entrepris de raviver la mémoire de certains pionniers de l'indépendantisme dans deux ouvrages récents : Leprophète solitaire: Raymond Barbeau et son époque, Montréal, Ordre naturiste social de Saint-Marc l'Évangéliste, 2000, 582 p., et (avec la collaboration spéciale de Jean Côté), Le patriote : Marcel Chaput et son époque, [Montréal], Ordre naturiste social de Saint-Marc l'Évangéliste, 2006, 606 p.

${ }^{13}$ André-J. Bélanger, Ruptures et constantes. Quatre idéologies du Québec en éclatement: La Relève, La JEC, Cité Libre, Parti pris, Montréal, Hurtubise HMH, 1977, 219 p. ; Pierrette Bouchard-Saint-Amant, «L'idéologie de la revue Parti pris : le nationalisme socialiste » in J.-P. Montminy et al., Idéologies au Canada français, 1940-1976. T. 1 : La presse - la littérature, Presses de l'Université Laval, 1981, p. 315-353 ; Malcolm Reid, The Shouting Signpainters; A Literary and Political Account of Quebec Revolutionary Nationalism, New York, Monthly Review Press, 1972, 315 p. Parmi les travaux les plus récents sur Parti pris et sur la pensée de la décolonisation québécoise, mentionnons les thèses de Mathieu Lavigne, L'idée de décolonisation québécoise. Le discours tiers-mondiste au Québec et sa quête identitaire (1963-1968), Mémoire de maîtrise (histoire), Université de Montréal, 2007, 259 f. ; de Sean Mills, The Empire Within: Montreal, the Sixties, and the Forging of a Radical Imagination, Thèse de doctorat (histoire), Queen's University (Kingston), 2007, vii, 432 f. ; et d'Alexis Lachaine, Black and Blue: French Canadian Writers, Decolonization and Revolutionary Nationalism in Quebec, 1960-1969, Thèse de doctorat (histoire), York University (Toronto), 2007, $311 \mathrm{f}$.

${ }^{1+}$ Le compte rendu le plus riche et le plus détaillé de ce mouvement est celui de Louis Fournier, FLQ. Histoire d'un mouvement clandestin, Outremont, Lanctôt éditeur, 1998 (édition revue et augmentée), 533 p. 
${ }^{15}$ Bien sûr, on a largement étudié d'autres personnages de transition, regroupés par l'historien Michael Behiels sous l'étiquette « néo-nationalistes » (principalement André Laurendeau et les historiens de «l'École de Montréal »), qui portèrent l'évolution du nationalisme de sa version traditionaliste à sa redéfinition moderniste: M. Behiels, Prelude to Quebec's Quiet Revolution: Liberalism versus Neo-nationalism, 1945-1960, Kingston et Montréal, McGillQueen's University Press, 1985, 366 p. Mais ceux-ci n'étaient pas ouvertement socialistes ni toujours très à gauche - qu'on pense à l'importance de la bourgeoisie pour l'École de Montréal -, et leur nationalisme resta en général en deçà de l'indépendantisme, du moins avant les années 1960.

${ }^{16}$ C'est à l'antinationalisme de l'équipe directrice de Cité libre (autour de Gérard Pelletier et Pierre Elliott Trudeau) que nous faisons ici référence, car on a souligné récemment la complexité de la question nationale au sein de cette revue, à laquelle ont collaboré plusieurs intellectuels qui deviendront indépendantistes dans les années 1960 (Fernand Dumont, Marcel Rioux, Pierre Vadeboncœur) : Martin Meunier et Jean-Philippe Warren, «De la question sociale à la question nationale : le cas de la revue Cité libre ", Recherches sociographiques, vol. 39, n"s 2-3 (1998), p. 291-316. Voir aussi Léon Dion, Québec, 1945-2000. T. 2. Les intellectuels et le temps de Duplessis, Québec, Presses de l'Université Laval, 1993, p. 280-283.

${ }^{17}$ Marcel Olscamp, Le fils du notaire : Jacques Ferron 1921-1949: genèse intellectuelle d'un écrivain, St-Laurent, Fides, 1997, 425 p. On assiste depuis quelques années à un vif regain d'intérêt pour l'œuvre (et le personnage) de Ferron : témoin la publication d'entretiens et de plusieurs recueils de textes, ainsi que la construction d'un site Web d'une richesse exceptionnelle (Jacques Ferron écrivain, http://www.ecrivain.net/ferron/).

18 "Roy, Raoul», dans Réginald Hamel et al., Dictionnaire des auteurs de langue française en Amérique du Nord. Montréal, Fides, 1989, p. 1198-1199; Magali Deleuze, L'une et l'autre indépendance : 1954-1964 : les médias an Québec et la guerre d'Algérie, Mont-Saint-Hilaire, Québec, Éditions Point de fuite, 2001, p. 101 ; Louis Fournier, FLQ..., p. 17.

1" Par exemple, Jacques Dumais, «Les cinq “pères” de l'indépendance. Un bon gouvernement qui a peur ", Le Soleil, 13 novembre 1978. Jacques Ferron, «Deux précurseurs ", L'information médicale et paramédicale, 169 (21 novembre 1978). Le militant nationaliste Gilles Rhéaume, qui a connu Roy, s'est efforcé de raviver le souvenir de ce dernier : « Raoul Roy, le penseur solitaire », La Presse québécoise, octobre 2005, p. 9 ; et « Raoul Roy, un pionnier méconnu du mouvement indépendantiste ", L'Action indépendantiste, Montréal, n" 19 (sep- 
tembre-octobre 2000), p. 11. Rhéaume semble également avoir été l'une des sources principales de l'intéressant portrait de Roy esquissé par J.-F. Nadeau dans son Bourgault, p. 83-85.

${ }^{20}$ Pour une analyse historique et théorique des points de convergence et de divergence possibles entre nationalisme et socialisme, voir Mathieu Lapointe, Nationalisme et socialisme dans la pensée de Raoul Roy, 1935-1965, Mémoire de maitrise (histoire), Université de Montréal, 2002, 212 f., introduction, section «Problématique ».

${ }^{21}$ Ce qui suit constitue en quelque sorte un condensé de notre mémoire de maîtrise consacré à Raoul Roy. Pour plus de détails sur la vie de Roy, son évolution idéologique et les institutions qu'il a fondées et animées jusqu'en 1965, voir Mathieu Lapointe, op. cit.

${ }^{22}$ Un certain nombre des renseignements biographiques sur la décennie 1930 sont tirés de quelques longues lettres adressées par Roy à Lionel Groulx de 1937 à 1939 : Fonds Lionel-Groulx (FLG), Archives du Centre de recherche Lionel-Groulx (ACRLG), Outremont (Québec), P1/A,3286. Nous les avons comparés avec divers documents du fonds Raoul Roy (FRR), ACRLG, fonds P64. Les lettres à Groulx sont également précieuses pour retracer l'évolution idéologique et psychologique de Roy pendant cette période. Pour une analyse approfondie de ces lettres, voir Mathieu Lapointe, «Entre ville et campagne, entre fascisme et communisme : les hésitations d'un jeune autodidacte beauceron, Raoul Roy (1914-1996) ", à paraitre dans les actes du colloque «1937: un tournant culturel ", dir. Yvan Lamonde et Denis Saint-Jacques, aux Presses de l'Université Laval (Coll. « Cultures québécoises »).

${ }^{23}$ Roy mentionnera à quelques reprises le manque de livres dans son milieu rural d'origine : voir par exemple l'entrevue de Raoul Roy par Marcel Chaput, 18 octobre 1976 (enregistrement sonore), Bibliothèque et Archives nationales du Québec (BAnQ), 535 avenue Viger Est, Montréal, Fonds Marcel-Chaput, P96, S1, P22. La formation scolaire de Roy, marquée par les interruptions, ne semble pas avoir dépassé la huitième année. À partir de 1941, date où il quitte le Séminaire des vocations tardives de Saint-Victor (Beauce), Roy poursuivra sa formation intellectuelle en autodidacte.

${ }^{24}$ Lettre de Raoul Roy à Lionel Groulx, St-Philibert, Québec, avril 1937, ACRLG, FLG, P1/A,3286.

${ }^{25}$ Transcription d'une lettre manuscrite de Raoul Roy à un ami (identité inconnue), Montréal, août 1935, ACRLG, FRR, P64/B1,2. 
26. Lettre de Raoul Roy à Lionel Groulx, St-Philibert, Québec, avril 1937, ACRLG, FLG, P1/A,3286.

${ }^{27}$ Sur La Nation, voir notamment Robert Comeau, « Les indépendantistes de La Nation et le séparatisme (1936-1938) ", Revue d'bistoire de l'Amérique française, vol. 26, n" 1 (juin 1972), p. 83-102; idem, Les indépendantistes québécois, 1936-1938, Thèse de maitrise (histoire), Université de Montréal, 1971, 2 volumes ; André-J. Bélanger, L'apolitisme des idéologies québécoises. Le grand tournant de 1934-1936, Québec, Presses de l'Université Laval, 1974, p. 329-352 ; et plus récemment, Charles-Philippe Courtois, Trois mouvements intellectuels québécois et leurs relations françaises: l'Action française, «La Relève » et "La Nation » (19171939), Thèse de doctorat (histoire), IEP-Paris / UQAM, 2007, xxv, 635 p.

${ }^{28}$ Lettre de Raoul Roy à Lionel Groulx, St-Philibert, Québec, avril 1937, ACRLG, FLG, P1/A,3286.

${ }^{29}$ Le fonds d'archives Lionel Groulx contient quatre lettres de Raoul Roy, datées respectivement d'avril 1937, du 24 mai 1937, du 5 décembre 1938 et du 24 février 1939 : ACRLG, FLG, P1/A,3286. Les réponses de Groulx sont malheureusement introuvables.

${ }^{30}$ Roy confie même à Groulx son rêve de fonder un mouvement qu'il appellerait «Jeunes Habitants » et qui, organisant l'expression du mécontentement paysan, serait « le plus grand mouvement de libération paysanne encore vu dans notre pays de Québec » : lettre de Raoul Roy à Lionel Groulx, SaintPhilibert, Québec, avril 1937, ACRLG, FLG, P1/A,3286. Ce projet demeurera lettre morte.

${ }^{31}$ Roy fait probablement allusion ici à la Guerre civile espagnole qui fait rage à cette époque et au cours de laquelle la presse québécoise rapportait de nombreux massacres de membres du clergé ; et peut-être aussi aux persécutions religieuses en URSS. Sur la couverture médiatique du conflit espagnol au Québec, voir Caroline Désy, "Si loin, si proche ». La Guerre civile espagnole et le Québec des années trente, [Sainte-Foy], CELAT et Presses de l'Université Laval, 2004, 177 p.

${ }^{32}$ Lettre de Raoul Roy à Lionel Groulx, St-Philibert, Québec, avril 1937, ACRLG, FLG, P1/A,3286, p. 9-11. Notre transcription des lettres respecte la ponctuation (ou son absence) employée par Roy.

${ }^{33}$ Lettre de Raoul Roy à Lionel Groulx, St-Philibert, Québec, 24 mai 1937, ACRLG, FLG, P1/A,3286, p. 2-3.

${ }^{34}$ Danièle Cuisinier, «Un journaliste nous vient des chantiers [Raoul Roy pose un bel exemple] », Combat, vol. 1, n" 25 (17 mai 1947). 
${ }^{35}$ Hewlett Johnson (1874-1966), The Socialist Sixth of the World, Londres, Gollancz, 1939, 384 p. Le titre devient The Soviet Powerà partir de 1940.

${ }^{36}$ Entrevue de Raoul Roy par Marcel Olscamp, Montréal, 25 septembre 1992, enregistrement sonore non publié. Nos remerciements à $\mathrm{M}$. Olscamp de nous avoir donné accès à ce document.

${ }^{37}$ Entrevue de Raoul Roy par Marcel Olscamp.

${ }^{3 *}$ «La crise de la presse canadienne », Combat (Montréal), 23 novembre 1946, p.1.

31) Pour ne donner que quelques titres d'articles, tous de rédacteurs anonymes : «Les Catholiques [sic] en Lithuanie soviétique » (24 mai 1947), « Ce fut la semaine du Vatican» (28 juin 1947) ou encore «Les communistes et le Vatican » (12 juillet 1947). Notre dépouillement de ce journal ne fut cependant pas systématique et il s'est limité aux années 1946-1949.

+" Pour une analyse idéologique du journal Searchlight, voir Robert Comeau et Jacques Samson, «Le Searchlight, journal exemplaire de l'Union des marins canadiens (1936-1949) ", Stratégie, n" 13-14 (été 1976), p. 7-47. Nous remercions le professeur Comeau d'avoir mis à notre disposition le journal Searchlight.

${ }^{+1}$ Pour la liste complète de ces articles de Roy à Combat et Searchlight, voir Mathieu Lapointe, Nationalisme et socialisme dans la pensée de Raoul Roy, annexe 2, p. $210-212$.

${ }^{+2}$ L'historienne Andrée Lévesque rapporte par exemple qu'en 1929, le Parti communiste du Canada reconnaissait que les Canadiens français constituaient « les masses les plus exploitées au Canada » : Virage à gauche interdit : les communistes, les socialistes et leurs ennemis au Québec, 1929-1939, Montréal, Boréal express, 1984, p. 46.

${ }^{43}$ Anonyme, «Peuple sans histoire ? ", Combat, 23 novembre 1946. Voir aussi « Hommage de COMBAT aux victimes de '37 », Combat, 7 décembre 1946, compte rendu d'une manifestation des "Amis de Combat", le 30 novembre 1946 au cimetière Côte-des-Neiges, pour célébrer le « souvenir des luttes démocratiques de notre histoire » et protester contre l'état piteux du monument à la mémoire des Patriotes. Dans une veine semblable, le $\mathrm{D}^{\mathrm{r}}$ Daniel Longpré appelle les professionnels à continuer de jouer leur rôle historique dans les « luttes de revendications » des Canadiens français : « Les professionnels dans la lutte actuelle », Combat, 17 mai 1947.

${ }^{*}$ Quelques années plus tôt, pendant la Guerre, le P.O.P. avait également privilégié l'unité nationale dans l'effort de guerre, aux dépens des désirs d'auto- 
nomie des Canadiens français. L'historien Robert Comeau cite une déclaration du congrès de 1943 : « Notre première préoccupation a toujours été, est et sera toujours de défendre les intérêts nationaux de notre pays, le Canada ». Il en conclut que " [l] direction communiste en vint à adopter des positions de plus en plus nationalistes canadiennes » : Comeau, «Le Parti communiste canadien, Stanley Bréhaut Ryerson et la question nationale canadienne-française, 1935-1965 ", dans Robert Comeau et Robert Tremblay (dir.), Stanley Brébaut Ryerson, un intellectuel de combat, Hull, Vents d'ouest, 1996, p. 145. Staline lui-même avait remis le patriotisme à l'honneur afin de gagner la Seconde Guerre mondiale (qu'on a appelée en URSS « la grande guerre patriotique ") : Hélène Carrère d'Encausse, Staline : l'ordre par la terreur, Paris, Flammarion, 1979, p. 131-150.

${ }^{45}$ Sur les luttes de ce syndicat, voir notamment Robert Comeau, « La Canadian Seamen's Union (1936-1949) : un chapitre de l'histoire du mouvement ouvrier canadien ", Revue d'histoire de l'Amérique française, vol. 29, n" 4 (mars 1976), p. 503-538; et William Kaplan, Everything That Floats: Pat Sullivan, Hal Banks, and the Seamen's Unions of Canada, Toronto, University of Toronto Press, $1987,241 \mathrm{p}$.

"'Anonyme, "Le député libéral de la Beauce se crée un marché aux esclaves ", Combat, 26 avril 1947 ; Raoul Roy, « La "charité chrétienne" à \$9 par semaine", Combat, 31 mai 1947 ; Rédacteur inconnu, «Le pharisien Ludger Dionne lance une offensive contre la classe ouvrière ", Combat, 21 juin 1947 ; Raoul Roy, " Des lettres de la Beauce qui condamnent Ludger Dionne », Combat, 28 juin 1947 ; idem, "Ainsi, Ludger Dionne aurait fait la charité !... », Combat, 2 août 1947 ; Rédacteur inconnu, « Pas d'augmentation chez Dionne », Combat, 30 août 1947 ; Daniel Longpré, « Le Canada - Un pays où fleurit l'esclavage - le "camp Dionne" ", Combat, 18 octobre 1947.

${ }^{47}$ Ajoutons que cela était probablement d'autant plus facile qu'il n'y occupait pas un poste important, et qu'il n'avait donc pas à faire sienne toute l'orthodoxie du Parti et à se prononcer sur des sujets délicats qui l'auraient forcé à choisir entre nationalisme et socialisme.

${ }^{48}$ Pour un exemple de ce refus et de sa vision du nationalisme, voir par exemple " Une lutte réelle de décolonisation. Entrevue avec Jacques Berque », La Revue socialiste, n" 7 (hiver 1963-1964), p. 5.

+" Combat (Montréal), vol.1, $\mathrm{n}^{\text {"s }} 8,9$ et 10 (18 janvier, 25 janvier et $1^{\text {cr février }}$ 1947). 
"Sette crise s'inscrit dans le contexte du début de la guerre froide et de la mise au pas par le Kominform des partis communistes nationaux. Ironie de l'histoire, c'est le militant et historien communiste Stanley B. Ryerson - futur partisan, lui aussi, de l'indépendance socialiste du Québec - qui fut alors chargé de venir de Toronto pour discipliner la section québécoise. Sur cette crise, voir surtout l'article de Bernard Dansereau, « Stanley Bréhaut Ryerson et les exclus de 1947 » dans Robert Comeau et Robert Tremblay (dir.), Stanley Bréhaut Ryerson, un intellectuel de combat, p. 105-130.

${ }^{51}$ Entrevue de Roy par Olscamp.

${ }^{52}$ Mathieu Lapointe, Nationalisme et socialisme..., p. 89-90.

${ }^{33}$ À l'époque de La Revue socialiste, Roy évitera tout commentaire qui pourrait donner à son mouvement des allures antisémites, et il s'en tiendra à une critique générale des « minorités satellites » du pouvoir " colonial anglo-saxon » qui domine selon lui le Québec. À la fin de la décennie suivante, il exprimera ses griefs plus ouvertement dans son livre Lettre aux Juifs de Montréal : le secret des Juifs, Montréal, Éditions du Franc-Canada, 1979. Voir aussi Raoul Roy, Socialisme! Lequel?, Montréal, Franc-Canada, 1976, p. 12-13; Entrevue de l'auteur avec Claire Roy Morissette et Anne Morissette, respectivement sœur et nièce de Roy, Montréal, 24 mai 2001.

${ }^{5+}$ Sur Raymond Barbeau et son mouvement, voir Éric Bouchard, Raymond Barbeau...

${ }^{5}$ Cette situation durera d'ailleurs plus de deux ans, jusqu'au lancement de $\mathrm{La}$ Rerue socialiste en avril 1959.

${ }^{56}$ Éric Bouchard rapporte que pour certains, « du simple fait d'avoir été présentée par Barbeau et associée au corporatisme, "l'idée d'indépendance perdait toute crédibilité et ne méritait pas qu'on s'y arrête sereinement" » : Pierre Gravel, cité par Bouchard, Raymond Barbeau..., p. 96.

${ }^{57}$ Raoul Roy, "Le nom de La Revue socialiste », manuscrit non publié, 12 pages, ACRGL, FRR, P64/A2,8. Voir aussi Jacques Ferron, Une amitié bien particulière, Montréal, Boréal, 1990, p. 99-100. Dans l'entrevue avec Marcel Olscamp, Roy rappelle en exemple la campagne qu'avait menée Barbeau contre Radio-Canada à la suite de la diffusion d'une émission controversée sur Marguerite d'Youville. Elle révélait selon lui le visage d'extrême droite « un peu farfelu » de l'Alliance, qui désirait rétablir la religion catholique à une époque où l'intelligentsia québécoise francophone en avait assez de l'Église.

${ }^{5 x}$ Sur cette ligue, voir Mathieu Lapointe, Nationalisme et socialisme..., p. 106111. 
${ }^{5 \%}$ Le « Nouveau Parti » était le nom courant du Nouveau Parti démocratique (fondé en août 1961) à ses débuts. Son implantation au Québec fut l'occasion de vifs débats portant notamment sur la question nationale et sur l'autonomie de la future section provinciale. L'année 1963 vit l'éclatement du parti naissant en un NPD-Québec fédéral et un Parti socialiste du Québec (P.S.Q.) provincial : sur ces questions, voir André Lamoureux, Le NPD et le Québec, 1958-1985, Montréal, Éditions du Parc, 1985, 230 p.

${ }^{(1)}$ Le tirage de $L a$ Revue socialiste semble avoir oscillé entre quelques centaines et un peu plus d'un millier d'exemplaires, alors qu'Éric Bouchard estime que celui de Laurentie a varié entre environ 1000 et 4000 exemplaires : op.cit., p. 71 ; Mathieu Lapointe, Nationalisme et socialisme..., p. 103-106. Parti pris aurait quant à elle tiré à 3500 exemplaires en 1964 : André Beaulieu et Jean Hamelin (dir.), La presse québécoise des origines à nos jours, Québec, Presses de l'Université Laval, t. 9 (1955-1963), p. 296.

${ }^{61}$ Luc Racine et Roch Denis soulignent, dans un article classique, cette importance de l'ASIQ dans la formation de jeunes militants. Selon eux, l'influence de l'ASIQ « ne doit quand même pas être sous-estimée : Des intellectuels de l'Université, des étudiants, ont fait leur premières armes théoriques en prenant connaissance des thèses de Raoul Roy »: «Histoire et idéologie du mouvement socialiste québécois (1960-1970) », Socialisme québécois, 21-22 (avril 1971), p. 55. Jean-François Nadeau abonde en ce sens et ajoute que le poète Gaston Miron serait devenu indépendantiste à la lecture de $L a$ Revue socialiste de Roy : Bourgault, p. 84.

62 " Une lutte réelle de décolonisation. Entrevue avec Jacques Berque », p. 5.

${ }^{63}$ Premièrement, les socialisations extensives appelées par La Revue socialiste ne pourraient manquer de violer le droit de propriété des « colonialistes anglosaxons ». Deuxièmement, Roy et d'autres rédacteurs de La Revue socialiste rêvent parfois de la fermeture ou de la confiscation "méritée » du Montreal Star et de la Gazette: Roger Beausoleil, "Jeunesse du socialisme », La Revue socialiste, n" 2, p. 26-28 ; Roy, "La Révolution de Cuba », La Revue socialiste, n" 4 (été 1960), p. 46. Enfin, le vaste programme d'unilinguisme français et de francisation (écoles, marché du travail, publicité, toponymie) que prône Roy porterait nécessairement atteinte aux droits linguistiques de la minorité anglophone tels qu'ils avaient été traditionnellement définis. La position de Roy au sujet de la légitimité de la violence politique est quant à elle complexe, changeante et souvent ambiguë : voir Mathieu Lapointe, Nationalisme et socialisme..., chapitre 3, section « Un nationalisme radical et intégral ». 
${ }^{(4)}$ Ibid., même section. Pour des exemples, voir Roy, « Québec et la démocratie ", La Revue socialiste, n" 1 (printemps 1959), p. 11 ; Jean-Paul D'Amours, "Mister Of Course ou le sens d'André Laurendeau » La Revue socialiste, n" 2 (automne 1959), p. 12 ; et Roy, "Le "Nouveau parti" sera autonomiste au Québec ou il ne sera pas! ", La Revue socialiste, n" 5 (printemps 1961), p. 51-52.

${ }^{\prime \prime}$ C'est par exemple l'image de Frank Scott que donne Jacques Ferron dans "Adieu au P.S.D. », La Revue socialiste, n" 4 (été 1960), p. 7-14.

"Raoul Roy, "Le R.I.N. face au colonialisme », La Revne socialiste, n"6 (automne 1962), p. 15-17. Sur ce point comme sur bien d'autres, la pensée de Roy est ambiguë parce qu'elle oscille entre modération et radicalisme : dans le dialogue intitulé "Socialisme, décolonisation et nationalisme... », un personnage incarnant le socialisme indépendantiste affirme à un endroit que la décolonisation "éliminera la minorité anglophone du Québec »: La Revue socialiste, $\mathrm{n}^{\text {" }}$ 8 (printemps 1965), p. 25. Cependant, il ne nous semble pas qu'il faille voir dans cette expression extrême un appel au meurtre politique.

${ }^{67}$ Maurice Dufort, « Les avatars de la direction syndicale De Hal Banks à Gérard Pelletier », La Revue socialiste, n" 2 (automne 1959), p. 13-25.

${ }^{68}$ Par exemple, un survol rapide de la revue Parti pris semble indiquer qu'on n'y mentionne presque jamais le nom de Roy ou de ses institutions, l'ASIQ et La Revue socialiste. Magali Deleuze a néanmoins repéré un texte où Paul Chamberland, l'un des piliers de Partipris, fait mention de ce devancier dans le cadre d'une rétrospective des cinq dernières années de lutte indépendantiste et affirme : "Nous devons à Roy l'usage des thèmes de décolonisation et de révolution ». Chamberland, «Bilan d'un combat », Partipris, septembre 1964, p. 22 ; cité par Deleuze, L'une et l'autre indépendance..., p. 101.

(9) Jacques Ferron, « Deux précurseurs ».

7" Yves Ménard, « Raoul Roy, 1914-1996. Pionnier du mouvement indépendantiste et penseur anticolonialiste ", texte non publié, Université de Montréal, 1999, p. 20. L'affirmation de Ménard porte d'abord sur les activités de Roy dans les années 1980, mais nous n'avons aucune raison de croire qu'il en allait autrement dans les années 1960. Nous remercions Monsieur Ménard d'avoir bien voulu nous communiquer ce texte et nous accorder quelques entretiens à propos de Roy.

${ }^{71}$ Facteur encore plus difficile à évaluer, la composition du groupe - par exemple la présence de jeunes radicaux intéressés par la violence - a pu elle aussi avoir un effet dissuasif. 
${ }^{72}$ Entrevue téléphonique d'André Major par Mathieu Lapointe, Montréal, 20 juin 2001. Major ne milita d'ailleurs pas très longtemps dans l'ASIQ.

${ }^{73}$ Sur cette conception, voir Raoul Roy, «L'Église et le socialisme décolonisateur ", L'indépendantiste, n" 2 (juin 1966), p. 16.

${ }^{7+}$ Voir, par exemple, Parti pris / j.m.p. [Jean-Marc Piotte], "Le socialisme ", Parti pris, n" 6 (mars 1964), p. 2-4. Voir également les reproches de Roy à ce sujet dans "Le succès de "Parti pris" et l'échec du MLP ", L'indépendantiste, $\mathrm{n}^{\text {" }}$ 2 (juin 1966), p. 4. Sur le caractère superficiel du marxisme de cette revue, voir André-J. Bélanger, Ruptures et constantes..., p. 165s et 192-193 ; et Jean-Marc Piotte - lui-même un membre fondateur de cette revue -, Un partipris politique. Essais, Montréal, VLB éditeur, 1979, p. 16 et 21 s.

${ }^{75}$ Ferron, « Deux précurseurs ».

${ }^{76}$ Malcolm Reid souligne ce fossé lorsqu'il présente Roy comme étant " [s]ympathetic to youth, but seing no relationship between beatniks and militants, poets and politicals » : The Shouting Signpainters..., p. 201.

${ }^{77}$ Roy, « Socialisme, décolonisation et nationalisme. À quelle révolution nous vouer ? ", La Revue socialiste, n" 8 (printemps 1965), p. 23-48.

${ }^{78}$ Lettre de Raoul Roy à des collaborateurs de La Revue socialiste, Montréal, 11 janvier 1964, ACRLG, FRR, P64/A5,1. Habitué de polémiquer contre la gauche fédéraliste et antinationaliste, Roy se montre prudent face à ce problème nouveau. Il écrit : «La position de RÉVOLUTION QUÉBÉCOISE est quand même ambiguë. On ne peut la qualifier de revue anti-indépendantiste ». Son attitude face à Vallières évoluera cependant très rapidement vers la colère et l'intransigeance.

${ }^{79}$ Roy, «Introduction ", Lindépendantiste, n" 2 (juin 1966), non paginé : [p. i] ; idem, "Le succès de "Parti pris" et l'échec du MLP ", in ibid., p. 4-7 ; idem, "Questions à "Parti pris" ", in ibid., p. 8.

${ }^{811}$ Louis Fournier, FLQ. Histoire d'un mouvement clandestin, Outremont, Lanctôt éditeur, 1998 (édition revue et augmentée), p. 113.

${ }^{81}$ Roy reviendra souvent sur le cas de l'attentat à l'usine de chaussures Lagrenade, le 5 mai 1966, qui tua une secrétaire canadienne-française, $\mathrm{M}^{\mathrm{me}}$ Thérèse Morin. Voir par exemple Roy, « F.L.Q. ou F.L.M. ? ", L'indépendantiste, $\mathrm{n}^{\prime \prime} 3$ (printemps 1968), p. 12 et 14.

${ }^{82}$ Ibid. Roy, "La maladie "valliérienne" de "Parti pris" et sa faillite idéologique » et " "Faire un Vietnam du Québec" », L’indépendantiste, n" 3 (printemps 1968), p. 6-8, 16-17. 
${ }^{83}$ Roy, « Des luttes à faire et à ne pas faire », L'indépendantiste, $\mathrm{n}$ " 2 (juin 1966), p. 9-10.

${ }^{8+}$ Roy, « La maladie "valliérienne”... », p. 6.

${ }^{\times 5}$ Il s'agissait de Gabriel Gagnon, « Pour un socialisme décolonisateur », Parti pris, vol. 4, n" 1 (septembre-octobre 1966), p. 40-56. Roy, "La maladie "valliérienne"... ", p. 6. Roy venait d'expliquer le sens de son socialisme dans "Le socialisme décolonisateur », L'indépendantiste, n"2 (juin 1966), p. 1-3.

" Roy, "Socialisme, décolonisation et nationalisme.... », p. 46. Idem, "Le socialisme décolonisateur», p. 1-3. Idem, "Pourquoi La Revue socialiste a été fondée ", L'indépendantiste, $\mathrm{n} " 3$ (printemps 1968), p. 9-11.

${ }^{87}$ Roy, Socialisme ! Lequel ? Montréal, Franc-Canada, 1976, 17 f. et p. 23-48.

${ }^{8 *}$ Roy, Marxisme : mépris des peuples colonisés ? Montréal, Éditions du FrancCanada, $1977,130 \mathrm{p}$.

${ }^{* \prime}$ Dans leur anthologie des manifestes québécois, Daniel Latouche et Diane Poliquin-Bourassa qualifiaient d'ailleurs le manifeste de La Revue socialiste (1959) de " point tournant » : « Par certains thèmes, il annonce les années ' 60 , par d'autres, il récapitule tous les manifestes antérieurs ». Dans la marge du manifeste, ils relevaient ensuite plusieurs curiosités, comme la persistance de « reliquats » du vocabulaire des années $1930:$ Le Manuel de la parole : manifestes québécois, t. 2 (1900 à 1959), Sillery, Éditions du Boréal express, 1977, p. 335347.

"Il est d'ailleurs intéressant de souligner ce fait peu connu que l'indépendantisme de Roy, comme celui de Raymond Barbeau, fut inspiré par le mouvement de Paul Bouchard et son journal La Nation. S'il s'avère que, comme l'affirme Robert Comeau, l'historien et «théoricien de l'indépendance » Maurice Séguin a lui aussi été inspiré par cet indépendantisme des années 1930, il y aurait matière à réévaluer l'importance de ce mouvement dans l'histoire de l'indépendantisme : voir notamment Robert Comeau, «Les séparatistes de 1936-1937. Paul Bouchard et La Nation ", Cap-aux-diamants, $\mathrm{n}^{\circ}$ 53 (printemps 1998), p. 26-29.

"Les marques de la formation et du parcours idéologiques de Roy dans son discours débordent certainement le domaine des idées. Au niveau du style, du vocabulaire et du ton, le discours de Roy portait la trace de plus de deux décennies de lectures idéologiques radicales, tant nationalistes que socialistes. 